\title{
PATOLOGIAS EM MATERIAIS NĀO CONVENCIONAIS: EXPANSIBILIDADE DA ESCÓRIA DE FORNO PANELA
}

\author{
FRACÃO DA SILVA, TAYNÁ \\ Engeheira Civil \\ Universidade Estadual de Campinas (UNICAMP) \\ São Paulo; Brasil \\ taynafracao@gmail.com \\ FERREIRA ROCHE PEREIRA, VANESSA \\ Tecnóloga em Construção Civil \\ Universidade Estadual de Campinas (UNICAMP) \\ São Paulo; Brasil. . \\ vanessarochee9@hotmail.com
}

\author{
C. DOS SANTOS FERREIRA, GISLEIVA \\ Professora \\ Universidade Estadual de Campinas (UNICAMP) \\ São Paulo; Brasil \\ gisleiva@ft.unicamp.br
}

\section{RESUMO}

A incorporação da escória de forno panela (EFP) em materiais cimentícios pode ser uma ótima alternativa para reduzir o passivo ambiental gerado pelo setor siderúrgico, além dos impactos ambientais causados pela indústria da construção civil. Entretanto, a composição química das amostras de EFP apresentam variações em função do método de resfriamento, o que pode proporcionar manifestações patológicas, por exemplo, a expansibilidade. Portanto, o objetivo desta pesquisa foi determinar a expansibilidade em pastas com adição ou substituição do cimento Portland $(\mathrm{CP})$ por EFP. Para isso, realizou-se ensaios de caracterização físico-química em três amostras de EFP (densidade, granulometria, DRX e FRX) e a determinação da expansibilidade em pastas cimentícias conforme ABNT NBR 11582:2016. A análise dos resultados indicou que as variações dimensionais das pastas com EFP encontraram-se dentro dos padrões permitidos pela ABNT NBR 16697:2018 $(\leq 5 \mathrm{~mm})$. Entre as pastas das três amostras de EFP e respectivos teores de adição e substituição de EFP estudados, verifica-se que a amostra A3 é mais estável do que as outras duas, sendo que as pastas A40 e 40M10 apresentaram porcentagens de expansibilidade iguais ao traço referência (0\% EFP). Com os resultados deste trabalho foi possível comprovar a sustentabilidade do uso da EFP em materiais cimentícios, além da redução dos efeitos expansivos deste resíduo, considerando o método de resfriamento e composição química da escória. Palavras-chave: resíduos sólidos, material cimentício, construção civil, expansibilidade, manifestação patológica.

\section{ABSTRACT}

The incorporation of ladle furnace slag (LFS) in cementitious materials can be a great alternative to reduce of the environmental liability generated by the steel industry, as well as reducing the environmental impacts caused by the construction sector. However, a chemical composition of LFS may exhibit changes due cooling method, which may cause pathological manifestations in this material, for example, expansibility. Therefore, the objective of this research was to determine the expansibility in cementitious pastes with addition or substitution of Portland cement by LFS. In order, was performed physico-chemical characterization tests of three LFS samples (specific mass, particle size, XRD and XRF) and the determination of the expansibility in cement pastes according to ABNT NBR 11582:2016. The analysis of the results indicated that the dimensional variations of the LFS pastes meet the requirements by ABNT NBR 16697: $2018(\leq 5 \mathrm{~mm})$. Between the pastes of the three LFS samples and their LFS addition and substitution contents studied, was verified that the sample A3 is more stable than the other two and the A40 and 40M10 pastes presented expansion percentages equal to the reference paste ( $0 \%$ LFS). The results of this research it was possible to prove the sustainability of the use of EFP in cementitious materials, in addition to reducing the expansive effects of this residue, considering the cooling method and chemical composition of the slag.

Keywords: solid waste, cementitious material, construction, expandability, pathological manifestation.

\section{INTRODUÇÃO}

Para atender os requisitos de sustentabilidade, o setor da construção civil tem buscado cada fez mais utilizar materiais não convencionais, principalmente aqueles com incorporação de resíduos gerados por outros setores produtivos, o que caracteriza vantagens recíprocas. Neste contexto está inserido o setor siderúrgico, tanto pela diversidade de resíduos 
como pelo volume gerado. Os vários tipos de escórias (de alto-forno, de aciaria, de forno panela) representam cerca de 21 milhões de toneladas anuais apenas no Brasil, ou seja, aproximadamente $607 \mathrm{~kg}$ de resíduo sólido a cada 1 ton de aço produzido (IABr, 2018). No âmbito internacional, os principais países geradores de escórias são os EUA com 19 milhões de toneladas (USGS, 2019); o Japão com 14 milhões (NSA, 2017); os países europeus com 21 milhões de toneladas (EUROSLAG, 2012) e a China com 100 milhões de toneladas (GUO, BAO, WANG, 2018).

A escória de forno panela (EFP), proveniente dos fornos de aciaria, atualmente é responsável por $27 \%$ dos resíduos sólidos gerados pela indústria siderúrgica (IABr, 2018). É importante destacar que a EFP é resultante do processo secundário de refino do aço para construção civil, que utiliza a sucata como principal matéria-prima, o que justifica a heterogeneidade dos lotes e, consequentemente, a necessidade de caracterização preliminar (RODRIGUEZ et al., 2009; ANASTASIOU, PAPAYIANNI, PAPACHRISTOFOROU, 2014; VILAPLANA et al., 2015).

Estes processos preconizam as diferenças relacionadas aos aspectos físicos (granulometria) e composição química (teores de óxido de cálcio $(\mathrm{CaO})$ e óxido de magnésio $(\mathrm{MgO})$ ), compostos responsáveis pelas reações químicas expansivas que podem ocorrer com este tipo de escória (BARBOSA, 2013). Outra particularidade é o processo de resfriamento utilizado por cada empresa (ao ar, por pelotização e brusco), o que também interfere na quantidade dos óxidos de cal e de magnésio de cada lote de EFP (FARIA, 2007). Portanto, o método de resfriamento é um fator fundamental para desencadear reações químicas indesejáveis entre a EFP e materiais cimentícios. (MASUERO, DAL MOLIN, VILELA, 2001; SETIÉN, HERNÁNDEZ, GONZÁLEZ, 2009; PELLEGRINO et al., 2013; VILAPLANA et al., 2015; HERRERO et al., 2016).

Segundo Pellegrino et al. (2013) os óxidos de cálcio se expandem, quando em contato com água, devido à rápida hidratação deste composto. Por outro lado, os óxidos de magnésio apresentam reações químicas de hidratação mais lentas, o que pode resultar na expansão tardia. Masuero et al. (2001) caracterizaram amostras de escórias e as aplicaram em argamassas como aglomerante, em substituição parcial do cimento Portland. Estes autores concluíram que o método brusco de resfriamento da EFP resulta em lotes com melhor qualidade, quando se pensa em utilizá-la como aglomerante.

A definição do teor ideal de EFP nos materiais cimentícios também pode limitar os problemas relacionados à expansibilidade. Setién et al. (2009) e Vilaplana et al. (2015) investigaram a incorporação de EFP como matéria-prima para a produção de cimento Portland e identificaram teores máximos distintos de EFP (20\% e 40\%, respectivamente).

Portanto, com foco na sustentabilidade e uso de materiais não convencionais na construção civil, este trabalho tem como objetivo principal obter a expansibilidade de pastas com adição de EFP ou substituição parcial do cimento Portland (CP) por EFP.

\section{METODOLOGIA}

\subsection{Materiais}

Nesta pesquisa foi possível estudar três amostras distintas de EFP, diferenciadas principalmente pelo método de resfriamento:

- A1: resfriamento ao ar;

- A2: resfriamento brusco;

- A3: resfriamento por pelotização.

A Tabela 1 apresenta a identificação e respectiva origem das três amostras de EFP e demais materiais utilizados na confecção das pastas utilizadas na pesquisa.

Tabela 1 - Principais materiais utilizados na confecção das pastas e respectivas origens.

\begin{tabular}{c|c|c|c}
\hline Material & Sigla & Origem/Tipo & Localização \\
\hline Água & - & Rede de abastecimento & Limeira, SP \\
\hline Cimento & CP V-ARI & Indústrias de cimento (Brasil) & Região de Limeira, SP \\
\hline Metacaulim & M-HP & Metacaulim do Brasil & Jundiaí, SP \\
\hline Escória de forno panela & A1 & & São Paulo \\
(EFP) & A2 & Indústria do setor siderúrgico & Espírito Santo \\
& A3 & & Minas Gerais \\
\hline
\end{tabular}

A preparação das amostras de EFP teve início com a secagem das mesmas em estufa a $105^{\circ} \mathrm{C}$ e posterior arrefecimento em temperatura ambiente. Devido à heterogeneidade granulométrica apresentada pelas amostras de EFP (Figura 1), 
adotou-se uma metodologia para o pré-processamento das amostras, conforme indicações de Papayianni e Anastasiou (2012):

a) separação da parcela retida na peneira com abertura de $1,18 \mathrm{~mm}$;

b) moagem da parcela passante na peneira com abertura de $1,18 \mathrm{~mm}$, utilizando moinho-de-bolas (ciclos de 15 min para cada $400 \mathrm{~g}$ de EFP).

O processamento das amostras foi realizado em moinho MA500 (velocidade fixada em +/- 200 RPM), jarro de cerâmica de alumina com capacidade de $1 \mathrm{~L}$ e 40 bolas cerâmicas de $20 \mathrm{~mm}$ de diâmetro. A Figura 2 ilustra as diferenças em relação à textura entre as três amostras de EFP (A1, A2 e A3), após as etapas “a e b” de pré-processamento, ou seja, peneiramento e moagem do material.

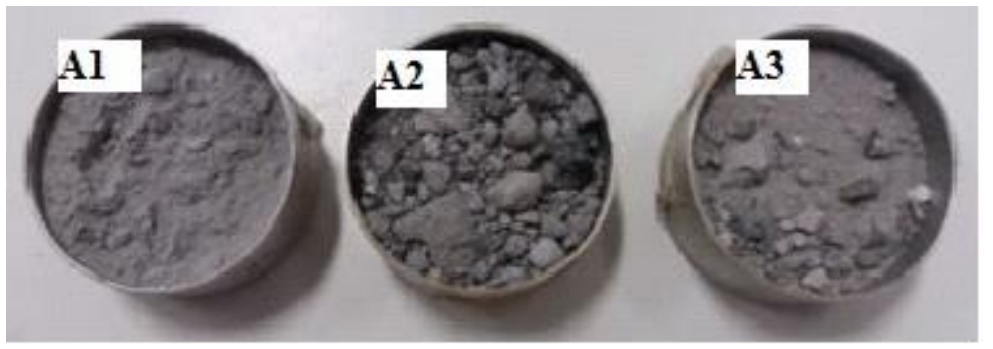

Figura 1: Amostras de EFP brutas, antes do processamento.

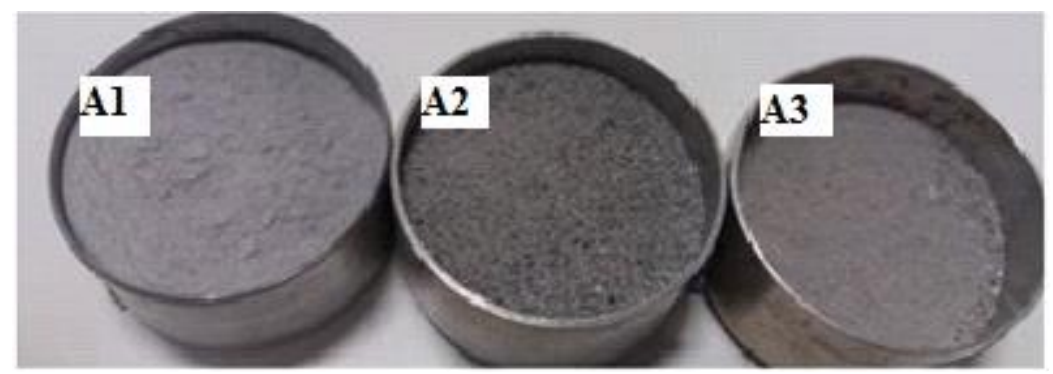

Figura 2: Amostras de EFP após as etapas de pré-processamento (peneiramento e moagem).

\subsection{Métodos}

A definição da metodologia deste trabalho teve como foco principal, identificar as propriedades físico-químicas de pastas cimentícias com EFP, definindo-se como parâmetro o teor de substituição e de adição da escória em relação ao consumo de cimento Portland. Os teores de 30, 40 e 50\% foram baseados em dados da literatura, onde o menor e o maior valor encontrado de substituição foi de $10 \%$ e $45 \%$, obtidos por Herrero et al. (2016) e Almeida (2017), respectivamente. Também optou-se por testar pastas de cimento Portland com EFP (teores de 30\%, 40\% e 50\%) e de metacaulim (10\%) para verificar o potencial deste resíduo em ativar materiais pozolânicos. As amostras de EFP foram submetidas a ensaios de densidade, granulometria, Difração de Raios X (DRX), Fluorescência de Raio X (FRX) e Perda ao fogo; já as pastas cimentícias ao ensaio de expansibilidade e obtenção de imagens de Microscopia eletrônica de varredura (MEV); todos executados conforme Tabela 2.

Tabela 2 - Ensaios de caracterização físico-química das amostras de EFP, metacaulim e pastas.

\begin{tabular}{c|c|c}
\hline & Norma & Objetivo \\
\hline Ensaio & Amostras de EFP e metacaulim \\
\hline Densidade & ABNT NBR 16605:2017 & Determinar a densidade da amostra \\
\hline Granulometria a laser & - & Granulometria e finura \\
\hline Difração de Raios X (DRX) & - & Composição (fases cristalinas e amorfas) \\
\hline Fluorescência de Raio X (FRX) & - & Composição química \\
\hline Perda ao fogo & - & Materiais orgânicos \\
\hline \multicolumn{2}{|c|}{ Pastas cimentícias } \\
\hline $\begin{array}{c}\text { Expansibilidade } \\
\text { varredura (MEV) }\end{array}$ & ABNT NBR 11582:2016 & Determinar a expansibilidade das pastas \\
\hline
\end{tabular}


Para medir a expansibilidade a frio foram moldados três corpos de prova para cada traço conforme procedimentos da ABNT NBR 16606:2017 e requisitos da ABNT NBR 11582:2016. Neste ensaio são utilizadas agulhas de Chateliê, as quais são colocadas sobre placas de vidros. A pasta é colocada até o enchimento total da agulha e em seguida é realizada a primeira medição da distância entre as hastes da agulha (a). Depois disso, as agulhas foram imersas em tanque com água potável (Figura 3) à temperatura $(23 \pm 2)^{\circ} \mathrm{C}$ e após sete dias realizou-se a segunda medição (b). A expansibilidade a frio é dada pela diferença entre as medições (b) e (a), em mm.

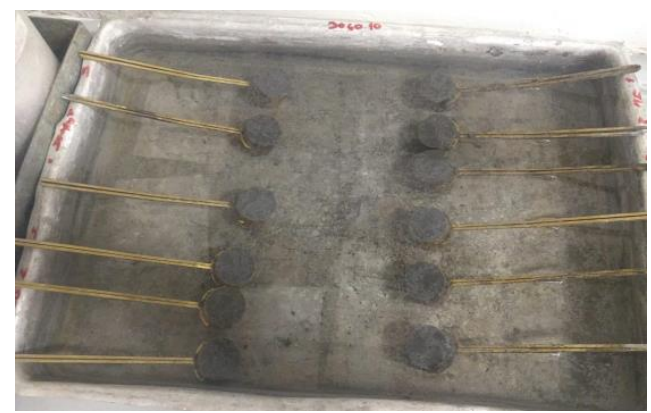

Figura 3: Agulhas de Chateliê com as pastas estudadas e mantidas imersas durante os ensaios de expansibilidade.

Amostras das pastas com EFP e pasta referência foram analisadas em Microscópio Eletrônico de Varredura (MEV) para se obter imagens da microestrutura. Estas imagens foram obtidas em equipamento MEV, modelo XL-30 FEG com Espectrômetro de Energia Dispersiva (EDS) acoplado, disponibilizado pelo Laboratório de Caracterização Estrutural do Departamento de Engenharia de Materiais da UFSCar, São Carlos, SP.

\section{RESULTADOS E DISCUSSÕES}

\subsection{Caracterização dos materiais}

A Tabela 3 apresenta a composição química das amostras de EFP e do metacaulim utilizados na pesquisa, obtidos conforme análises de FRX. Para efeito de comparação, foram extraídos da literatura os resultados da composição química de amostras de EFP e do CP, de trabalhos que utilizaram a mesma análise.

Tabela 3 - Composição química das amostras de EFP, cimento Portland e metacaulim.

\begin{tabular}{|c|c|c|c|c|c|c|c|c|}
\hline \multirow{2}{*}{$\begin{array}{c}\text { Compostos } \\
\text { Químicos (\%) }\end{array}$} & \multicolumn{3}{|c|}{ EFP } & \multirow{2}{*}{$\begin{array}{l}\text { Marinho } \\
\text { et al. } \\
(2017)\end{array}$} & \multirow{2}{*}{$\begin{array}{l}\text { Herrero } \\
\text { et al. } \\
(2016)\end{array}$} & \multirow{2}{*}{$\begin{array}{l}\text { Sétien } \\
\text { et al. } \\
(2009)\end{array}$} & \multirow[b]{2}{*}{ Metacaulim } & \multirow{2}{*}{$\begin{array}{c}\text { Rojas et } \\
\text { al. } \\
(2013) \\
\text { Cimento }\end{array}$} \\
\hline & A1 & A2 & A3 & & & & & \\
\hline $\mathrm{CaO}$ & 54,20 & 35,60 & 52,30 & $\begin{array}{c}57,30- \\
64,40 \\
\end{array}$ & $\begin{array}{c}53,96- \\
61,64 \\
\end{array}$ & $\begin{array}{c}50,00- \\
58,00 \\
\end{array}$ & 0,3 & 63,9 \\
\hline $\mathrm{Fe}_{2} \mathrm{O}_{3}$ & 3,40 & 26,80 & 14,50 & $\begin{array}{c}0,70- \\
4,60 \\
\end{array}$ & $\begin{array}{c}3,82- \\
1,01 \\
\end{array}$ & $\begin{array}{l}1,60- \\
3,30 \\
\end{array}$ & 2,0 & 2,99 \\
\hline $\mathrm{SiO}_{2}$ & 11,50 & 18,00 & 16,50 & $\begin{array}{c}24,50- \\
36,80\end{array}$ & $\begin{array}{l}3,77- \\
22,94 \\
\end{array}$ & $\begin{array}{l}12,00- \\
20,00 \\
\end{array}$ & 58,2 & 19,6 \\
\hline $\mathrm{MgO}$ & 8,80 & 8,03 & 6,82 & $\begin{array}{l}6,20- \\
8,40\end{array}$ & $\begin{array}{c}5,99- \\
6,36 \\
\end{array}$ & $\begin{array}{l}7,50- \\
12,00 \\
\end{array}$ & 0,4 & 0,73 \\
\hline $\mathrm{MnO}$ & 2,68 & 1,89 & 1,12 & $\begin{array}{c}0,20- \\
1,60\end{array}$ & $\begin{array}{c}0,22- \\
0,29 \\
\end{array}$ & $\begin{array}{c}0,36- \\
0,52\end{array}$ & - & - \\
\hline $\mathrm{Al}_{2} \mathrm{O}_{3}$ & 1,32 & 4,13 & 3,28 & $\begin{array}{c}1,00- \\
3,90\end{array}$ & $\begin{array}{l}4,42- \\
28,82 \\
\end{array}$ & $\begin{array}{l}4,00- \\
19,00 \\
\end{array}$ & 32,8 & 5,34 \\
\hline $\mathrm{SO}_{3}$ & 1,93 & 2,11 & 1,78 & - & $\begin{array}{c}2,80- \\
3,43 \\
\end{array}$ & - & 3,2 & 3,44 \\
\hline $\mathrm{F}$ & 0,59 & 1,52 & 1,93 & - & - & - & - & - \\
\hline $\mathrm{Cr}_{2} \mathrm{O}_{3}$ & 0,06 & 0,71 & 0,43 & $\begin{array}{c}0,40- \\
1,40 \\
\end{array}$ & - & $\begin{array}{c}0,01- \\
0,10\end{array}$ & - & - \\
\hline $\mathrm{TiO}_{2}$ & 0,29 & 0,46 & 0,6 & - & $\begin{array}{c}0,14- \\
0,34\end{array}$ & $\begin{array}{c}0,33- \\
0,89 \\
\end{array}$ & 1,2 & - \\
\hline
\end{tabular}




\begin{tabular}{c|c|c|c|c|c|c|c|c}
\hline $\mathrm{ZnO}$ & 0,10 & 0,07 & 0,24 & - & - & - & - & - \\
\hline $\mathrm{P}_{2} \mathrm{O}_{5}$ & 0,09 & 0,23 & 0,19 & $\begin{array}{c}0,20- \\
0,30\end{array}$ & $\begin{array}{c}0,04- \\
0,01\end{array}$ & - & - & - \\
\hline $\mathrm{SrO}$ & 0,13 & 0,12 & 0,17 & - & - & - & - & - \\
\hline $\mathrm{Na}_{2} \mathrm{O}$ & 0,09 & 0,08 & 0,13 & - & - & $1,60-2,4$ & 0,1 & 0,09 \\
\hline $\mathrm{K}_{2} \mathrm{O}$ & 0,11 & 0,05 & 0,12 & - & - & - & 1,7 & 0,83 \\
\hline $\mathrm{BaO}$ & 0,09 & 0,10 & 0,07 & - & - & - & - & - \\
\hline $\mathrm{Cl}$ & 0,09 & 0,03 & 0,03 & - & - & - & - & - \\
\hline $\mathrm{ZrO}$ & 0,03 & 0,03 & 0,02 & - & - & - & - & - \\
\hline $\mathrm{PF}(\%)$ & 14,50 & $\mathrm{GF}$ & $\mathrm{GF}$ & - & $\begin{array}{c}26,2 \mathrm{PF}- \\
3 \mathrm{GF}\end{array}$ & - & - & 2,90 \\
\hline $\mathrm{CaO} / \mathrm{Al}_{2} \mathrm{O}_{3}+\mathrm{SiO}_{2}$ & 4,22 & 1,60 & 2,64 & - & - & $\begin{array}{c}1,60- \\
2,40\end{array}$ & - & - \\
\hline
\end{tabular}

Foi verificado que os compostos químicos das escórias de forno panela e suas quantidades são semelhantes entre si. As diferenças podem ser atribuídas principalmente pela heterogeneidade dos lotes de sucata, a principal matéria-prima empregada na fabricação do aço e, consequentemente, na geração de EFP. Os principais compostos são os óxidos de cálcio, de silíca, de ferro e de magnésio, que representam praticamente $78 \%, 88 \%$ e $90 \%$ da massa total das amostras 1 , 2 e 3 , respectivamente.

A amostra A1 apresentou perda ao fogo de 14,5\%, já as amostras A2 e A3, ao contrário, apresentaram ganho de massa, ou seja, ganho ao fogo (GF) quando expostas à temperatura de $1200^{\circ} \mathrm{C}$. O ganho ao fogo pode ser atribuído à oxidação de alguns minerais presentes nas amostras. Lemos (2010) verificou um aumento de massa na amostra de EFP devido à oxidação do enxofre, já na pesquisa de Bravo e Silva (2017) também ocorreu o GF, mas devido à oxidação de metais como o alumínio e ferro. Nas amostras A2 e A3, que apresentaram GF verifica-se a presença de $26,8 \%$ e $14,5 \%$ do composto $\mathrm{Fe}_{2} \mathrm{O}_{3}$, respectivamente, enquanto na amostra A1, que apresentou PF, apenas 3,4\%, Portanto atribui-se o ganho ao fogo das amostras 2 e 3 ao elevado teor de ferro $\left(\mathrm{Fe}_{2} \mathrm{O}_{3}\right)$.

Diversos autores relatam que a presença de óxidos de magnésio e de cálcio pode apresentar reações químicas expansivas na presença da água, o que pode afetar a estabilidade física da matriz cimentícia (FALESCHINI et al., 2015; PELLEGRINO et al., 2013; VILAPLANA et al., 2015; YILDIRIM e PREZZI, 2017). O óxido de cálcio em contato com água se hidrata rapidamente, o que resulta em compostos expansíveis, enquanto o óxido de magnésio reage de forma mais lenta, resultando em expansão tardia (PELLEGRINO et al., 2013). Ao comparar o teor de CaO, verifica-se que as amostras A1 e A3 apresentaram praticamente o mesmo teor (54\% e 52\%, respectivamente), teores que podem ser considerados altos quando comparados com a amostra A2 (35\%). Entretanto, os autores pesquisados utilizaram amostras de EFP com teores entre 50 e $60 \%$ de $\mathrm{CaO}$, os quais são coerentes com as amostras A1 e A2. Mas também é importante verificar o teor de $\mathrm{MgO}$, que ficou entre 7 e $9 \%$ nas 3 amostras desta pesquisa, o que corrobora os valores de Marinho et al. (2017) e Herrero et al. (2016). Portanto, o método de resfriamento dos lotes de EFP é um fator definitivo para o uso desta escória em materiais cimentícios, sabendo-se que o resfriamento brusco e a pelotização provocam a hidratação do óxido de cálcio, formando o hidróxido de cálcio, composto mais estável. Isto pode ser comprovado pelos resultados das análises de DRX, onde foi possível identificar os principais compostos cristalinos presentes nas amostras de EFP, através da identificação dos picos presentes nos respectivos difratogramas (Figura 4).

Os principais óxidos presentes nas amostras de EFP $\left(\mathrm{CaO}, \mathrm{SiO}_{2}, \mathrm{Fe}_{2} \mathrm{O}_{3}, \mathrm{AlO}_{2}, \mathrm{MgO}\right)$ encontram-se combinados, formando os compostos minerais identificados na Tabela 4, tanto para as 3 amostras de EFP desta pesquisa como de outros autores pesquisados (MARINHO et al., 2017; HERRERO et al., 2016; SETIÉN et al., 2009). A partir de uma análise minunciosa, foi possível identificar os principais minerais em comum (Silicato de Cálcio, Portlandita, Calcita, Quartzo, Periclásio e Cuspidina).

A amostra 3 apresenta picos com maior intensidade de compostos resultantes da hidratação do $\mathrm{CaO}$ (portlandite) e da sua combinação com outros óxidos (silicatos de cálcio e cuspidina). Isto indica um menor potencial para desencadear reações expansivas, portanto, mais indicada para a incorporação em matrizes cimentícias. Por outro lado, verifica-se picos intensos de periclásio na amostra 3, o que pode ser controlado com adições minerais ou pozolânicas (GAMEIRO et al., 2014). 


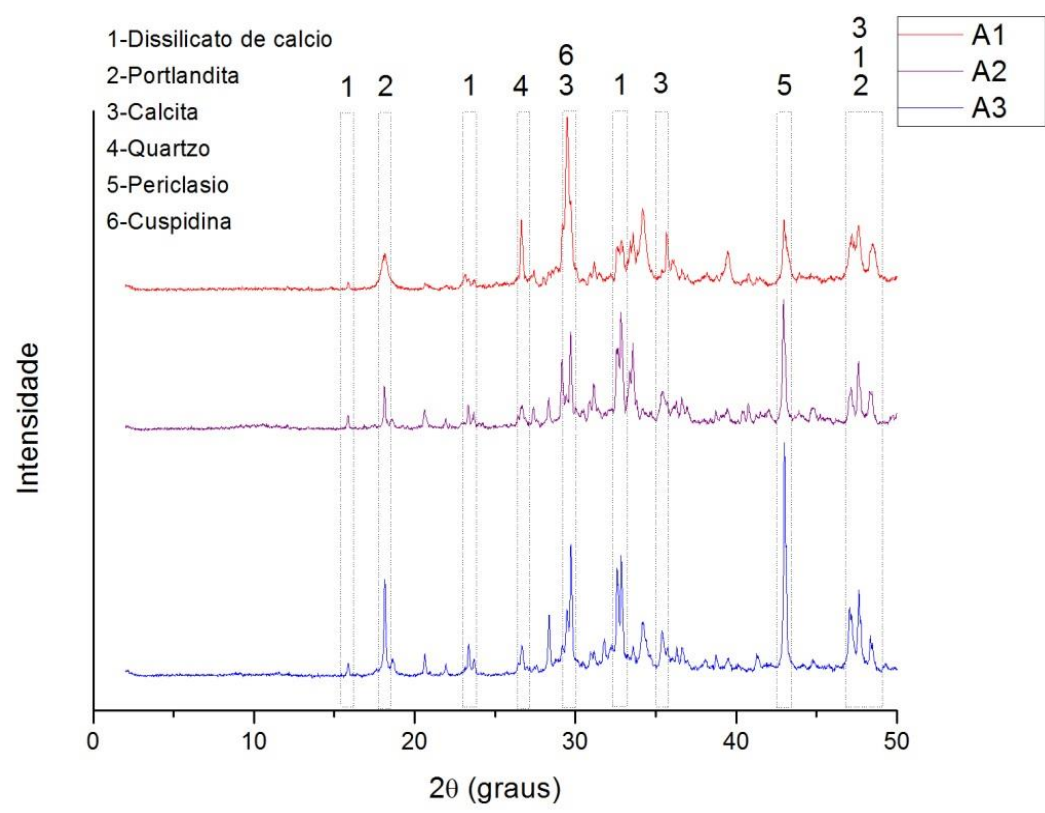

Figura 4: Difratograma comparativo das amostras de EFP.

Tabela 4 - Composição mineralógica por DRX.

\begin{tabular}{c|c|c|c|c|c|c|c}
\hline Compostos (\%) & Nomenclatura & $\mathrm{A} 1$ & $\mathrm{~A} 2$ & $\mathrm{~A} 3$ & $\begin{array}{c}\text { Marinho } \\
\text { et al. } \\
(2017)\end{array}$ & $\begin{array}{c}\text { Herrero } \\
\text { et al. } \\
(2016)\end{array}$ & $\begin{array}{c}\text { Sétien } \\
\text { et al. } \\
(2009)\end{array}$ \\
\hline $\mathrm{Ca}_{2}\left(\mathrm{SiO}_{4}\right)$ & Silicato de cálcio & $\mathrm{X}$ & $\mathrm{X}$ & $\mathrm{X}$ & $\mathrm{X}$ & $\mathrm{X}$ & $\mathrm{X}$ \\
\hline $\mathrm{Ca}(\mathrm{OH})_{2}$ & Portlandita & $\mathrm{X}$ & $\mathrm{X}$ & $\mathrm{X}$ & $\mathrm{X}$ & & \\
\hline $\mathrm{CaCO}_{3}$ & Calcita & $\mathrm{X}$ & $\mathrm{X}$ & $\mathrm{X}$ & $\mathrm{X}$ & & \\
\hline $\mathrm{SiO}_{2}$ & Quartzo & $\mathrm{X}$ & $\mathrm{X}$ & $\mathrm{X}$ & $\mathrm{X}$ & & \\
\hline $\mathrm{MgO}_{\mathrm{Ca}_{4} \mathrm{Si}_{2} \mathrm{O}_{7} \mathrm{~F}_{2}}$ & Periclásio & $\mathrm{X}$ & $\mathrm{X}$ & $\mathrm{X}$ & $\mathrm{X}$ & $\mathrm{X}$ & $\mathrm{X}$ \\
\hline $\mathrm{Fe}_{3} \mathrm{O}_{4}$ & Magnetina & $\mathrm{X}$ & $\mathrm{X}$ & $\mathrm{X}$ & & & \\
\hline $\mathrm{CaF}_{2}$ & Fluorita & & $\mathrm{X}$ & $\mathrm{X}$ & $\mathrm{X}$ & $\mathrm{X}$ & $\mathrm{X}$ \\
\hline $\mathrm{Mg}_{(\mathrm{OH})_{2}}$ & Brucita & & $\mathrm{X}$ & $\mathrm{X}$ & $\mathrm{X}$ & $\mathrm{X}$ & $\mathrm{X}$ \\
\hline $\mathrm{Ca}_{3} \mathrm{Mg}_{2}\left(\mathrm{SiO}_{4}\right)_{2}$ & Merwinita & $\mathrm{X}$ & $\mathrm{X}$ & & $\mathrm{X}$ & $\mathrm{X}$ & $\mathrm{X}$ \\
\hline $\mathrm{Ca}_{3}\left(\mathrm{SiO}_{4}\right) \mathrm{O}$ & Silicato de cálcio & & & $\mathrm{X}$ & & $\mathrm{X}$ & $\mathrm{X}$ \\
\hline
\end{tabular}

A Tabela 5 apresenta os valores de massa específica das amostras de EFP (A1, A2 e A3), do cimento Portland e metacaulim (conforme dados fornecidos pelos respectivos fabricantes). Verifica-se que os valores obtidos para as três amostras de EFP são próximos entre si, exceto a A1. Ao comparar os valores das escórias com aqueles apresentados pelos demais materiais (CP e metacaulim), verifica-se que as amostras A2 e A3 apresentaram valores similares ao do CP V-ARI (aglomerante hidráulico) e a amostra A1 ao do Metacaulim (material pozolânico). Os valores obtidos por outros autores, também apresentados na Tabela 5, indicam heterogeneidade entre os valores dos índices físicos das amostras de EFP. Esta variação pode ser justificada pelo tempo de armazenamento (HERRERO et al., 2016), tipo de resfriamento e composição química dos lotes da escória (SETIÉN, HERNÁNDEZ e GONZÁLEZ, 2009; YI et al., 2012).

Tabela 5 - Massa específica dos materiais.

\begin{tabular}{|c|c|c|c|c|c|c|c|c|}
\hline \multirow[b]{2}{*}{ Ensaio } & \multicolumn{6}{|c|}{ EFP } & \multicolumn{2}{|c|}{ Aglomerantes } \\
\hline & A1 & A2 & A3 & $\begin{array}{c}\text { Yi et } \\
\text { al. } \\
(2012)\end{array}$ & $\begin{array}{c}\text { Marinho } \\
(2015)\end{array}$ & $\begin{array}{c}\text { Herrero } \\
\text { et al. } \\
(2016)\end{array}$ & $\mathrm{CP}$ & Metacaulim \\
\hline $\begin{array}{c}\text { Massa } \\
\text { específica } \\
\left(\mathrm{g} / \mathrm{cm}^{3}\right)\end{array}$ & 2,76 & 3,07 & 2,98 & $\begin{array}{c}3,0 \mathrm{a} \\
3,6\end{array}$ & 2,83 & 2,75 & 3,12 & 2,53 \\
\hline
\end{tabular}


As curvas granulométricas obtidas para as três amostras de EFP (método de Granulometria a laser) estão dispostas na Figura 5, juntamente com a curva do metacaulim.

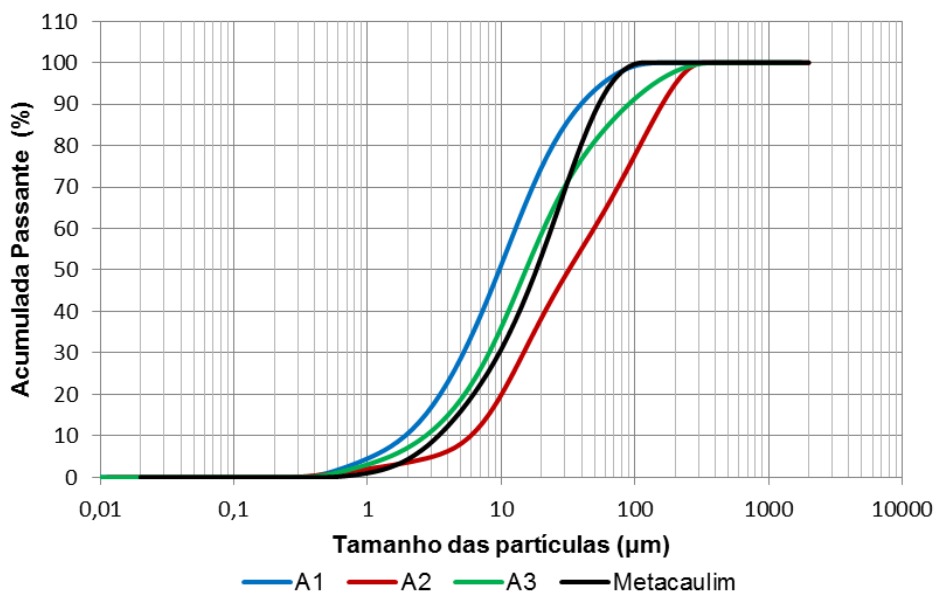

Figura 5: Curva granulométrica das amostras de EFP e do metacaulim.

Os principais dados extraídos da Figura 5 estão apresentados na Tabela 6 (diâmetro médio (DM); diâmetro correspondente a $10 \%$ do material passante $(\mathrm{D}(0.1))$; diâmetro correspondente a $50 \%$ do material passante $(\mathrm{D}(0.5))$ e diâmetro correspondente a $90 \%$ do material passante $(\mathrm{D}(0.9))$.

Tabela 6 - Características granulométricas das amostras de EFP, amostra de metacaulim, cimento Portland (fornecidas pelo fabricante) e de autores pesquisados (SETIÉN et al., 2009; PAPAYIANNI e ANASTASIOU, 2012).

\begin{tabular}{c|c|c|c|c}
\hline & \multicolumn{4}{|c}{ Unidade $(\mu \mathrm{m})$} \\
\hline & $\mathrm{D}(0.1)$ & $\mathrm{D}(0.5)$ & $\mathrm{D}(0.9)$ & Diâmetro médio \\
\hline $\mathrm{A} 1$ & 2,15 & 93,73 & 44,57 & 18,46 \\
\hline $\mathrm{A} 2$ & 2,53 & 66,79 & 165,10 & 52,40 \\
\hline $\mathrm{A} 3$ & 3,07 & 81,51 & 101,65 & 35,22 \\
\hline Metacaulim & - & - & - & 12,04 \\
\hline Cimento CP V ARI (fabricante) & 2,22 & 14,06 & 36,54 & 22,50 \\
\hline Setién et al. (2009) & 3,00 & - & 55 & - \\
\hline Papayianni e Anastasiou (2012) & $3,5-10$ & $70-90$ & $20-150$ & - \\
\hline
\end{tabular}

D (0.1) - Diâmetro correspondente a $10 \%$ do material passante.

D (0.5) - Diâmetro correspondente a $50 \%$ do material passante.

D (0.9) - Diâmetro correspondente a 90 \% do material passante.

A amostra que apresentou a maior finura foi a $\mathrm{A} 1 \mathrm{DM}=18,5 \mathrm{~mm})$, valor próximo aos apresentados pelo $\mathrm{CP}$ e metacaulim $(\mathrm{DM}=22,5 \mathrm{~mm}$ e $\mathrm{DM}=12,5 \mathrm{~mm})$, respectivamente. Ao comparar a finura das três amostras com a literatura, verifica-se que as escórias estudadas são mais finas do que aquelas utilizadas por Setién et al. (2009) e Papayianni \& Anastasiou (2012), o que melhor qualifica as 3 amostras de EFP, quando o objetivo é uitliza-las como aglomerante. É importante destacar que a amostra A3 é a mais viável entre as 3 escórias, se considerarmos a composição química, menor teor de $\mathrm{CaO}$ e $\mathrm{MgO}$, além do diâmetro médio das partículas $(35 \mu \mathrm{m})$, praticamente igual ao diâmetro correspondente a $90 \%$ do material passante do cimento Portland $(35,5 \mu \mathrm{m})$.

\subsection{Resultados da determinação da expansibilidade nas pastas cimentícias}

Na Tabela 7 são apresentados os valores médios de expansibilidade das pastas de cimento com EFP (A1; A2; A3) após 7 dias de ensaios. A ABNT NBR 16697:2018 estabelece que a expansibilidade do Cimento Portland com ou sem adições seja menor que $5 \mathrm{~mm}$. A maior expansibilidade apresentada pelas pastas estudadas neste trabalho foi de $3,7 \mathrm{~mm}$ (A1S40 e A2S50), portanto, todas as pastas atendem o valor máximo de expansibilidade indicado pela norma citada. Também são apresentados os valores do desvio padrão (DP), P-Valor e do coeficiente de variação (CV), obtidos em análise estatística realizada através do software Statgraphics Centurion (versão XVI). O P-valor deve ser $\leq 0,05$ para que os dados dos ensaios sejam considerados válidos, o que não foi possível para a amostra A1. É importane salientar 
que a metodologia adotada para determinar a expansibilidade das pastas infere algumas dificuldades de execução, o que justifica os altos valores de DP e CV.

Tabela 7 - Resultados de expansibilidade média $(\mathrm{mm})$ da pasta referência e também das pastas com EFP (A1; A2; A3), considerando 1 dia e 7 dias de cura, e dados estatísticos (P-valor; DP; CV).

\begin{tabular}{c|c|c|c|c}
\hline Amostras & Média $(\mathrm{mm})$ & DP $(\mathrm{mm})$ & P-Valor & CV $(\%)$ \\
\hline Referência & 1,30 & - & - & - \\
\hline A1 & 2,60 & 1.19 & 0.29 & 45.78 \\
\hline A2 & 2,08 & 1.32 & 0.04 & 63.48 \\
\hline A3 & 2,36 & 1.11 & 0.02 & 47.18 \\
\hline
\end{tabular}

Com a mesma análise estatística foi possível verificar que a expansibilidade apresentada pelas pastas está dentro do mesmo grupo homogêneo (Figura 6). Os grupos homogêneos são determinados por uma comparação múltipla entre cada uma das médias de cada variável em relação às demais, sendo que não há diferenças estatisticamente significativas entre os traços que compartilham o mesmo grupo. Portanto, esta análise nos leva a concluir que as variações numéricas da expansibilidade entre as 3 amostras de EFP não são estatisticamente significativas, ou seja, são iguais.

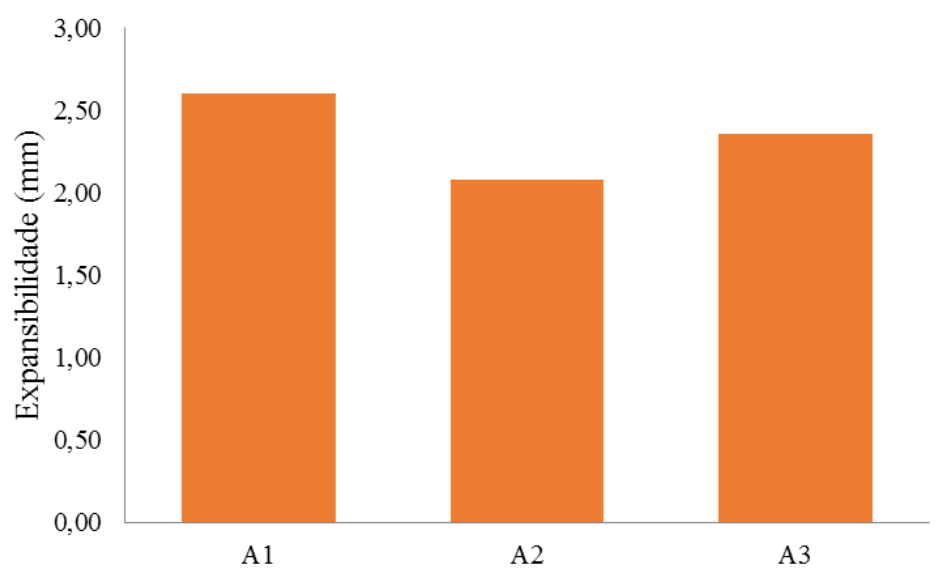

Figura 6: Expansibilidade das pastas com escória de forno panela (EFP).

FARIA (2007) utilizou a mesma norma para determinar a expansibilidade de pastas cimentícias com EFP, obtendo valor médio de 3,3 mm para as pastas com adição de $40 \%$ de escória e 1,7 mm para a pasta referência. Ao comparar os resultados desse autor com as pastas com mesmo teor de EFP (A40), é possível concluir que as 3 amostras estudadas apresentam melhor comportamento a este tipo de patologia.

Uma análise mais criteriosa indicou que as pastas A30, S30, 30M10 e S50 são estatisticamente iguais, assim como a pasta referência e a A40. Isso ocorreu porque o metacaulim presente na pasta 40M10 proporcionou um equilíbrio entre os teores de cal hidratada e o consumo deste composto pelas reações pozolânicas, o que corrobora as conclusões listadas por Gameiro et al. (2014) e Herrero et al. (2016).

Para estudos futuros, deve-se considerar outros métodos para se obter a expansibilidade das pastas ou até mesmo da amostra de EFP. Isso porque os óxidos predominantes neste tipo de escória $(\mathrm{CaO})$ se expandem quando em contato com água (rápida hidratação) e o óxido de magnésio apresenta expansão tardia. Portanto, a metodologia adotada deve incluir idades mais avançadas para a determinação da expansibilidade e assim obter resultados mais confiáveis para se determinar o desempenho e a durabilidade de materiais cimentícios com EFP (ROJAS e ROJAS, 2004).

Para ilustrar o melhor comportamento das pastas com metacaulim tem-se as imagens apresentadas na Figura 7, obtidas com MEV. Os compostos portlandita e etringita foram identificados mais facilmente nas pastas S30. Nas pastas com $10 \%$ de metacaulim, foi mais difícil visualizar tais compostos, principalmente na amostra 40M10. Esse comportamento pode ser justificado pelo consumo da portlandita durante as reações pozolânicas, que ocorrem devido a presença do metacaulim. 

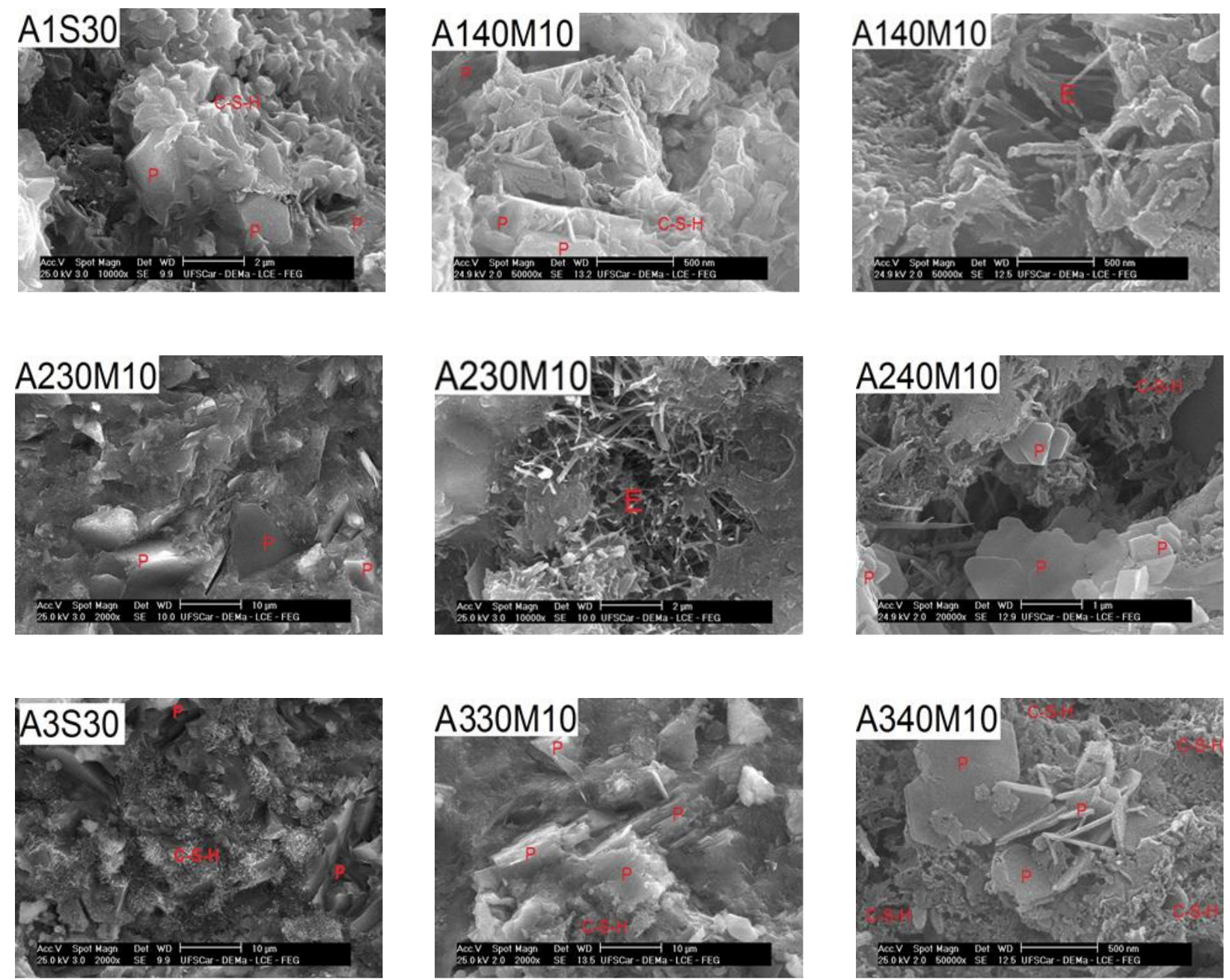

Figura 7: Microscopia das pastas com identificação de Portlandita (P).

Portanto, o uso de materiais não convencionais nas construções pode ser sustentável desde que ocorra uma análise criteriosa dos lotes de matérias primas não convencionais, aliada a uma segregação e armazenamento ideal.

\section{CONCLUSÕES}

Os resultados obtidos neste trabalho inferiram as seguintes conclusões:

As pastas apresentaram valores crescentes de expansibilidade em função da amostra de EFP (A1>A3>A2) no período avaliado (7 dias). Entretanto, ao se considerar o conjunto dos resultados apresentados, conclui-se que a amostra A3 é a mais indicada como adição mineral ou substituição ao cimento Portland devido à finura e composição química, o que contribui para um comportamento mais estável.

Ao considerar os requisitos da ABNT NBR 11582:2016 conclui-se que as 3 amostras de EFP atendem o valor máximo de expansibilidade $(5 \mathrm{~mm})$. Mas é importante obter esse parâmetro para idades mais avançadas devido presença do composto expansivo periclásio, que pode resultar na formação tardia de etringita.

Para próximas pesquisas, destaca-se a importância da caracterização de cada lote de EFP antes de sua aplicação, pois apresentam heterogeneidades em relação aos aspectos físico-químicos. Estas características podem ser justificadas pelos processos que cada indústria adota nas etapas de produção, retirada, segregação e armazenamento da escória, além da matéria-prima utilizada, que podem influenciar diretamente na qualidade da escória.

\section{REFERÊNCIAS}

ASSOCIAÇÃO BRASILEIRA DE NORMAS TÉCNICAS. NBR 11582: Cimento Portland - Determinação da expansibilidade Le Chatelier. Rio de Janeiro, 2016. 
ASSOCIAÇÃO BRASILEIRA DE NORMAS TÉCNICAS. NBR 16605: Cimento Portland e outros materiais em pó - Determinação da massa específica. Rio de Janeiro, 2017.

ASSOCIAÇÃO BRASILEIRA DE NORMAS TÉCNICAS. NBR 16606: Cimento Portland - Determinação da pasta de consistência normal. Rio de Janeiro, 2017.

ASSOCIAÇÃO BRASILEIRA DE NORMAS TÉCNICAS. NBR 16697: Cimento Portland - Requisitos. Rio de Janeiro, 2018.

ALMEIDA, M. C. A. Powder de Escória de Aciaria em Concreto de Alto Desempenho. Monografia (Graduação em Engenharia Civil). Universidade Federal de Ouro Preto, Ouro Preto, 2017.

ANASTASIOU, E. K.; PAPAYIANNI, I.; PAPACHRISTOFOROU, M. Behavior of self compacting concrete containing ladle furnace slag and steel fiber reinforcement. Materials \& Design, v. 59, p. 454-460, 2014.

BARBOSA, M. R. Caracterização geoambiental da escória de aciaria de cinco estados brasileiros. [s.l.] Dissertação (Mestrado em Geotecnia). Universidade Federal de Ouro Preto, Ouro Preto, 139f, 2013.

BRAVO, L. F. R.; SILVA, E. R. DA. Influência do tempo e da temperatura na oxidação dos aços SAE 1020 E SAE 304. Monografia (Graduação em Engenharia Mecânica). Universidade de Rio Verde, Rio Verde, 11p, 2017.

EUROSLAG. Statistics. 2012. Disponível em: < https://www.euroslag.com/products/statistics/statistics-2012/>. Acesso em Acesso em: 18 nov. 2019.

FALESCHINI, F. et al. High performance concrete with electric arc furnace slag as aggregate: Mechanical and durability properties. Construction and Building Materials, v. 101, p. 113-121, 2015.

FARIA, R. A. F. Contribuição ao estudo das propriedades mecânicas e da durabilidade de concretos com escória de aciaria eletrica (EAF) como agregado graúdo. [s.l.] Dissertação (Mestrado em Engenharia Civil). Universidade Federal de Góias, Goiânia,146p, 2007.

GAMEIRO, A. et al. Physical and chemical assessment of lime-metakaolin mortars: Influence of binder: aggregate ratio. Cement and Concrete Composites, v. 45, p. 264-271, 2014.

GUO, J.; BAO, Y.; WANG, M. Steel slag in China: Treatment, recycling, and management. Waste Management, v. 78, p. 318-330, 2018.

HERRERO, T. et al. Effect of high-alumina ladle furnace slag as cement substitution in masonry mortars. Construction and Building Materials, v. 123, p. 404-413, 2016.

INSTITUTO AÇO BRASIL. Aço e Sustentabilidade. 2018. Disponível em: < http://www.acobrasil.org.br/site/portugues/biblioteca/Folder_Sustentabilidade_externo_2018.pdf >. Acesso em: 18 nov. 2019.

INSTITUTO AÇO BRASIL. Relatório de Sustentabilidade. 2018. Disponível em: < http://www.acobrasil.org.br/sustentabilidade/>. Acesso em: 18 nov. 2019.

LEMOS, B. R. S. Oxidação de sulfetos promovida por materiais carbonáceos ativados. Dissertação (Mestrado em Química Inorgânica). Departamento de Quimica do Instituto de Ciências Exatas, Universidade Federeal de Minas Gerais, Belo Horizonte, 67f, 2010.

MARINHO, A. L. B. Aglomerante Ecológico Para Argamassa - Reciclagem da Escória de Forno Panela. Dissertação (Mestrado em Engenharia Civil). Escola de Minas, Universidade Federal de Ouro Preto, Ouro Preto, 115f, 2015.

MASUERO, A. B.; DAL MOLIN, D. C. C.; VILELA, A. C. F. Estabilização das escórias de aciaria elétrica com vistas a sua utilização como substituição ao cimento. Ambiente Construído, v. 4, n. 2, p. 57-81, 2001. 
NIPPON SLAG ASSOCIATION. Production and Uses of Steel Slag in Japan. Disponível em: < slg.jp/pdf/Steel\%20Slag\%202017FY\%20rev.pdf>. Acesso em: 20 nov. 2019.

PAPAYIANNI, I.; ANASTASIOU, E. Effect of granulometry on cementitious properties of ladle furnace slag. Cement and Concrete Composites, v. 34, n. 3, p. 400-407, 2012.

PELLEGRINO, C. et al. Properties of concretes with black/oxidizing electric arc furnace slag aggregate. Cement and Concrete Composites, v. 37, n. 1, p. 232-240, 2013.

RODRIGUEZ, Á. et al. Strength and workability of masonry mortars manufactured with ladle furnace slag. Resources, Conservation and Recycling, v. 53, n. 11, p. 645-651, 2009.

ROJAS, C.M.; CINCOTTO, M.A. Influência da estrutura molecular dos policarboxilatos na hidratação do cimento Portland. Ambiente Construído, v. 13, n. 3, p. 267-283, 2013.

ROJAS, M. F.; ROJAS, M. I. S. Chemical assessment of the electric arc furnace slag as construction material: Expansive compounds. Cement and Concrete Research, v. 34, n. 10, p. 1881-1888, 2004.

SCIENCE FOR A CHANGING WORLD. Iron and Steel Slag. Disponível em: < https://prd-wret.s3-us-west2.amazonaws.com/assets/palladium/production/atoms/files/mcs-2019-fesla.pdf>. Acesso em: 17 nov. 2019.

SETIÉN, J.; HERNÁNDEZ, D.; GONZÁLEZ, J. J. Characterization of ladle furnace basic slag for use as a construction material. Construction and Building Materials, v. 23, n. 5, p. 1788-1794, 2009.

VILAPLANA, A. S.-D.-G. et al. Utilization of Ladle Furnace slag from a steelwork for laboratory scale production of Portland cement. Construction and Building Materials, v. 94, p. 837-843, 2015.

YI, H. et al. An Overview of Utilization of Steel Slag. Procedia Environmental Sciences, v. 16, p. 791-801, 2012.

YILDIRIM, I. Z.; PREZZI, M. Experimental evaluation of EAF ladle steel slag as a geo-fill material: mineralogical, physical e mechanical properties. Construction and Building Materials, v. 154, p. 23-33, 2017. 